\title{
Etiology and Gene Mutation
}

\begin{abstract}
The external ear is composed of the auricle and the external auditory canal and develops from the first and second branchial arches.

The etiology of an external ear anomaly is considered to involve direct and indirect inhibition of the modulation of expression or function of the genes involved in morphogenesis of the external ear at an early stage of gestation period (the first trimester). Microtia may be caused by monogenic mutation, or abnormalities in the gene expression site or the mechanism of gene expression regulation due to chromosomal translocation. Familial recessive heredity of microtia was reported to be due to mutation of the HOXA1 gene which plays an important role in morphogenesis of the second brachial arch. Microtia and atresia of the external auditory canal are observed in patients which chromosomal aberrations of four types of trisomy $(13,18,21,22$ chromosomes) and three types of chromosomal deletions $(5 p-, 18 p-, 18 q-)$. On the other hand, it is argued to be induced by environmental factors or multifactorial heredity rather than monogenic heredity.
\end{abstract}

